\title{
Drugs Supply and Laboratory Services in Dots System of Kaduna State: A Health Care Worker Perspective
}

\author{
Shehu Usman Adamu1 ${ }^{*}$, D. McGill² \\ ${ }^{1}$ Biological Sciences, Kaduna State University, Kaduna, Nigeria \\ ${ }^{2}$ University of Liverpool, Liverpool, UK \\ Email: *usmanmodibbo@yahoo.com
}

How to cite this paper: Adamu, S.U. and McGill, D. (2018) Drugs Supply and Laboratory Services in Dots System of Kaduna State: A Health Care Worker Perspective. Journal of Tuberculosis Research, 6, 19-35. https://doi.org/10.4236/jtr.2018.61003

Received: February 15, 2017

Accepted: March 4, 2018

Published: March 7, 2018

Copyright $\odot 2018$ by authors and Scientific Research Publishing Inc. This work is licensed under the Creative Commons Attribution-NonCommercial International License (CC BY-NC 4.0). http://creativecommons.org/licenses/by-nc/4.0/ (c) (i) (9) Open Access

\begin{abstract}
A study was conducted in Kaduna State Nigeria to ascertain the impact of Drug Supply and laboratory services towards effective functioning of DOTS system. Six DOTS providing centres were identified for the study and in each centre, three respondents were selected based on their profession, the Medical Officer, the Pharmacist and the Laboratory Technician totaling 18. The officers were interviewed for the purpose of the research. Semi structured Interviews were conducted in this research as a means of obtaining Health workers perspective. The Interview which was conducted in their respective work station was open ended and all questions are same for all respondents. During the course of the study, all respondents agreed that the sources of drugs supply to their respective centres was the Kaduna State Tuberculosis and leprosy control Program office from the state capital and that drugs are been supplied quarterly and are adequate with slight interruption due to Logistic while for Laboratory services the respondents were of the view that there is the need to improve on it as there are cases of shortage of reagents and erratic supply of Electricity for effective Laboratory functioning.
\end{abstract}

\section{Keywords}

Drug Supply and Laboratory Services, Workers Perspective

\section{Introduction}

Tuberculosis, an infectious bacterial disease caused by Mycobacterium tuberculosis, remains world's most leading cause of death from a single infectious agent [1]. The disease is cosmopolitan that registered its presence in every single human 
society in the world with different degree of severity since $4000 \mathrm{BC}$. The fight against tuberculosis has been on since 1882 when Robert coach Identified Mycobacterium tuberculosis as the causative agent [2]. Mycobacterium tuberculosis causes the disease and is the leading cause of death due to single infectious agent [3], and majority of infected people are from developing countries, including Nigeria. According to World health Organization, Nigeria was fourth in the world in terms of number of people infected [4] with turberculosis widely spread and the leading cause of mortality in the country [5]. The disease is considered the disease of the poor and poverty affects over $80 \%$ of Nigerians. This indicates that ttuberculosis is certainly going to be a major health problem in developing countries [4] [6]. Despite the development of strong antibiotics, the incidence of turberculosis has been on the increase and was considered a Global Health problem by the World Health Organization. The cases of co-infection of tuberculosis with HIV/AIDS in Nigeria were reported among other countries [7].

Tuberculosis is known to be the disease of the poor the world over and most of the mortality due to the disease is known to be higher among the poor and in developing countries [4] [8]. The socio economic conditions associated with poverty such as poor housing, overcrowding and malnutrition are known to be factors responsible for spread of the disease. Survey of TB patients has shown that most are found in impoverish areas of the world and are mostly afraid of discrimination [9]. Similarly, the poorest and the most vulnerable were found to have least access to treatment even where treatment is offered free [10].

Tuberculosis (TB) control has been one of the major challenges facing developing countries for decades. The Nigerian Health system has integrated TB control into various aspects of social systems such as Primary Health care delivery, children are vaccinated against Tuberculosis. In order to ensure successful treatment and effective control of tuberculosis, several methods such as pill counts, combination therapy and Hospitalization and supervised therapy have been developed to ensure patient compliance with treatment. However, the results were not as promising and led to the development and introduction of Direct Observed Therapy Short course (DOTS) in 1994 [3]. DOTS as a TB control measure was designed with four cardinal principles [1] diagnosis and follow up through sputum microscopy [2], regular and uninterrupted supply of drugs [3] accurate cohort recording and analysis, and [4] direct observation of treatment by people responsible for health services. In its efforts to bring the disease under control the Nigerian government has adopted DOTS control strategy in 23 States and the Federal Capital Territory.

Kaduna is one of the 36 States of Nigeria and has adopted DOTS strategy in its 23 Local Government Councils Hospitals and some private clinics [11]. The effective control of TB through the use of DOTS in Kaduna Statewere associated with problems such as shortage of funds, low literacy level and poor report of TB cases [4] [12]. Similarly, there are many cases of Tuberculosis that are undetected despite large number of DOTS agents in the Local Government Areas of 
the state [13]. If the programme is to succeed, the bottle neck has to be identified and the perspectives of the health workers delivering the services of DOTS need to thouroughly understood. The current study was designed to review literature on TB control using DOTS and its effectiveness in TB control; to explore the perspective of health workers on the effectiveness of DOTS in Tuberculosis control in Kaduna State; to undertake thematic analysis and to make recommendations on ways to improve DOTS and effective TB control in Kaduna State.

\subsection{Aims and Objectives of the Study}

The major Aim of the research includes among others is to explore the perspectives of health workers on Drugs Supply and laboratory services both on the effectiveness of DOTS in TB control and how to improve the system in order to find the best possible ways of improving the DOTS in Kaduna State in order to make recommendations to Kaduna State Ministry of Health and the State Agency for Leprosy and Tuberculosis control. Besides the research question and aim, the present study has the following objectives

1) To explore the perspectives of health workers on Drugs supply and Laboratory services on the effectiveness of DOTS towards Tuberculosis control in Kaduna state, through 18 semi structured interviews.

2) To analyse data using thematic analysis in order to identify themes.

3) To make recommendations to Kaduna State Ministry of Health on how to improve DOTS through effective drugs supply and Laboratory services towards effective TB control in Kaduna State.

The outcome of the study is a comprehensive data on the major challenges facing the DOTS program in Kaduna state and suggested ways through which it can be improved. It is the first study of its kind that used to the perspective of health workers to come up with ways through which the Tuberculosis control program can be improved.

\subsection{Positionality}

Researcher is a lecturer with the Kaduna State University and was member of the Kaduna State House of Assembly and one time chairman House committee of Health; this has not been disclosed to the respondents as it may influence their answers to the questions. The researcher have an insight into the Kaduna State Ministry of health and disease control programs and this has helped during the identification of sites and understanding some of the responses of the respondents' particularly with regards to Government efforts.

\section{Methodology}

\subsection{Study Design}

The research was conducted using qualitative methods which enable the researcher to understand how the DOTS could be improved from the health workers' perspective and to understand why the effectiveness of DOTS is reduced. 
Qualitative method is a form of research aimed at understanding the social reality of individual, people, group or culture through exploration of their behavior, perspective feelings or experience [14]. The research method also allowed for data to be generated through experience of the health workers answering the "Why" and "How" on the DOTS services [15]. Qualitative research allows for exploration of individuals' perspectives and was better suited for the study as such information cannot be obtained using the quantitative study technique [16]. The choice of qualitative method for the research was not at the researcher discretion rather because of the nature of the research question, and the fact that it allowed for exploration of value, meanings, beliefs, thoughts, experience and feelings of the phenomenon under investigation [17] [18].

\subsection{Setting of the Study Area}

Kaduna State Lies in North-Western part of Nigeria between latitude $09002^{\prime} \mathrm{N}$ and 110 32'S and between longitude 960 15'E and 080 60'E. The State has an estimated population of 6.1 million people [19] distributed in its 23 Local Government Areas divided into three geo-political zones, the Northern (8 Local Governments), the Central (7 LGA) and the Southern (8 LGA) with socio-cultural differences between the northern part of the state where Hausa-Fulani Moslems predominate and the southern part of the State where six other ethnic groups, mainly Christians reside [11]. Agriculture in form of crop production and animal husbandry is the major occupation of the populace of Kaduna state. A great percentage of the people (70\%) are poor living below the poverty level which makes them prone to Tuberculosis and other Tropical diseases with high incidence of malaria. The health care structure of the state consist of about 961 Primary health care centres which are operated by the 23 Local Government councils; secondary health care facilities and tertiary level Hospitals are operated by the state Government. The State is also blessed with a University Teaching hospital at Shika and a Federal centre of tuberculosis control at Saye both in Zaria metropolis.

There is a DOTS centre in each of the 23 Local Governments with some Local Governments hosting secondary Health facility with more than one DOTS centre. Similarly, the Local Governments of Urban centres have DOTS centres in some private Hospitals. Tuberculosis control activity in Nigeria is centrally coordinated by the National Leprosy and Tuberculosis control Agency. All states of the Federation have a state office of Leprosy and TB control program which coordinates and control all DOTS centres in the Local Government areas. In Kaduna State, the Agency is headed by a Director under the Ministry of Health.

\subsection{Sampling Approach}

The sampling was purposeful and subjects were selected based on their functions and experience in the DOTS program [20] [21] [22]. Two DOTS centres were selected from each of the 3 Geographical zones of the state at random using the 
lottery Method. Names of centres from each Zone were written on a piece of paper, after thorough mixing, two papers were picked; the same was repeated for each Geo political zone to arrive at the 6 DOTS centres.

\subsubsection{Sample Inclusion/Exclusion}

Adult Health workers who are directly involved in the provision of DOTS services in the health centres were included in the research while other health workers that are not DOTS services providers were excluded for the research. In each centre, a DOTS Medical Officer, a Pharmacist and a Laboratory technician were selected for the research due to their direct involvement in DOTS service provision. DOTS service providers were included because of their experience in DOTS service provision and the possibilities of obtaining qualitative data based on their experience [20] [23].

\subsubsection{Sample Size}

Six DOTS providing centres were selected, two each from one of the geographical zones. The centres were Ikara and Zaria from Northern senatorial zone, Do$\mathrm{ka}$ and Makera from Central zone and Kachia and Sanga from the Southern senatorial Zone. In each centre, three respondents were interviewed based on their profession. The Medical Officer, the Pharmacist and the Laboratory Technician were selected. Table 1 below shows demographics on the 18 respondents.

Table 1. Demographics on the 18 respondents.

\begin{tabular}{|c|c|c|c|c|c|}
\hline S/No & Code & Area & Age & Sex & Role \\
\hline 1 & $\mathrm{Z1R} 1$ & Zone 1 Ikara & 48 & Male & DOTS drugs dispensing \\
\hline 2 & $\mathrm{Z} 1 \mathrm{R} 2$ & Zone 1 Ikara & & Male & Lab Technician \\
\hline 3 & Z1R3 & Zone 1 Ikara & & Male & DOTS supervisor(Medical \\
\hline 4 & Z1R4 & Zone 1 Zaria & & Male & Pharmacist \\
\hline 5 & Z1R5 & Zone 1 Zaria & & Male & Medical Doctor DOTS \\
\hline 6 & Z1R6 & Zone 1 Zaria & & Male & Lab Technician \\
\hline 7 & Z2R1 & Zone2 Makera & & Female & DOTS Dispensary \\
\hline 8 & $\mathrm{Z} 2 \mathrm{R} 2$ & Zone2 Makera & & Male & Lab Technician \\
\hline 9 & Z2R3 & Zone2 Makera & & & \\
\hline 10 & Z2R4 & Zone2 Doka & & Male & Lab Technician \\
\hline 11 & Z2R5 & Zone2 Doka & & Male & DOTS Supervisor \\
\hline 12 & Z2R6 & Zone2 Doka & & Female & DOTS Dispensary \\
\hline 13 & $\mathrm{Z} 3 \mathrm{R} 1$ & Zone3 Kachia & & Male & DOTS Supervisor \\
\hline 14 & $\mathrm{Z} 3 \mathrm{R} 2$ & Zone3 Sanga & & Male & Lab Technician \\
\hline 15 & Z3R3 & Zone3 Sanga & & Male & DOTS Dispensary \\
\hline 16 & $\mathrm{Z} 3 \mathrm{R} 4$ & Zone3 Sanga & & Male & DOTS Supervisor Medical \\
\hline 17 & Z3R5 & Zone3 Kachia & & & DOTS Dispensary \\
\hline 18 & Z3R6 & Zone3 Kachia & & & Lab Technician \\
\hline
\end{tabular}

$\mathrm{Z} 1$ is the code for respondents from zone 1 comprising of Zaria and Ikara; We therefore have respondents Z1R1, Z1R2, Z1R3, Z1R4, Z1R5 and Z1R6. Z2 is the code for respondents from zone 2 comprising of Doka and Makera; We therefore have respondents Z2R1, Z2R2, Z2R3, Z2R4, Z2R5 and Z2R6. Z3 is the code for respondents from zone 3 comprising of Kachia and Sanga; We therefore have respondents Z3R1, Z3R2, Z3R3, Z3R4, Z3R5 and Z3R6. 


\subsubsection{Sample Recruitment}

Eighteen Adult health workers in the DOTS centres were recruited for the research. Respondents were recruited based on personal contact at their respective clinics by the researcher and with the help of a recruitment assistant who is a DOTS service provider but not partaking in the research and who only served as a recruiter. The recruitment assistant was trained on ethical issues requiring confidentiality. The recruitment assistant went round the randomly selected centres and discussed the study with the potential respondents about the research. Participants Information sheet (PIS) was given to Potential respondents by the recruiter which has all the information about the research and their rights, similarly, they also given consent form which they ticked as appropriate and signed. Consent was verified with the respondent during interview. One of respondents declined to be interviewed after reading the PIS and signing the consent form on the account that he didn't want his voice recorded. He agreed to note taking, He was subsequently dropped from the research and replaced by another DOTS service provider from the same clinic.

\subsection{Data Collection Method}

\subsubsection{Pilot Interview}

A pilot interview was conducted in accordance with [24] methods which based on the pilot outcome, questions were retained with some increase in follow up questions and the ways of asking questions was strengthened to always ask for further explanation. The pilot interview was transcribed and responses indicated shying away from answering Government commitment towards Tuberculosis control. The aspect of Government commitment was further stressed or asked as a follow up question thereafter in subsequent interviews.

\subsubsection{Main Interview}

Eighteen (18) Adult health workers were interviewed for the purpose of the research. Semi structured Interviews were conducted in this research as a means of obtaining Health workers perspective. The Interview was open ended and all questions are same for all respondents [25] and participants were allowed to answer the questions in their own way or style and were further asked "Why" and "How" to further enrich the data, similarly, other particular areas were pursued in more depth due to response from respondent in accordance with methods of [26]. Questions in most cases were reframed or reconstructed to allow for easy understanding by respondent; this is done in order to allow respondents fully express their views and experiences [27]. Interviews were recorded with a Sony MP3 digital Recorder model ICDUX80. Each Interview was conducted at the respective work place of the respondents which are either Laboratory, medical office where consultations with patients' takes place or dispensaries unit where drugs and ledgers are kept to ensure a relaxed atmosphere [28] to enable them have high level of confidence and in accordance with best interview practice of [29]. Interviews were conducted in English and respondents were allowed 
to express themselves in their own ways. While taping the interviews, key notes of important points were jotted to complement voice data. Respondents were coded to ensure anonymity. Main topical questions asked during the interview in order to explore the perspective of the health workers to the effectiveness of DOTS towards tuberculosis control in Kaduna state focused on detection of new cases, availability of drugs, effectiveness of Laboratory services, major problems hindering the performance of the centre and their possible solutions as well as views on Government commitments and how best to improve the DOTS services. Other follow up questions were asked since the interview was open ended.

Similarly, the records of Interview, transcript and field notes and analysis were done to increase the credibility of the research which will increase its validity as well [30].

\subsection{Ethical Consideration}

Ethics, which is defined by Markkula Centre for Applied Ethics as well, based standards of right and wrong that prescribe what humans ought to do, usually in terms of right, obligations, benefit to society, fairness, or specific virtues [31] is adopted for the purpose of this research. In every qualitative research, ethics is very important as the research subjects are human beings that are protected by Universal Human right declaration and right to be respected and other local laws as the research settings may require.

Ethical considerations relating to qualitative research that involves the responsibility of the researcher to the respondent participants such as Informed consent, protection of participants from harm or risk associated with the research, anonymity of the respondents to ensure confidentiality, reciprocity and Feedback of the research findings were ensured [32] [33] [34] [35].

\subsection{Analytical Approach}

\subsubsection{Transcription}

The recorded digital data was transferred to a computer windows media file. Interviews were later transcribed from the computer by the researcher to allow for listening to the data over and over for easier analysis. Transcription was made in accordance of the methods described by [36], where a combination of verbatim transcription of content recorded voice and researchers notation of participants' nonverbal behavior recorded during the interview was used to increase reliability, validity and veracity of data collection [37] [38].

Results were compared to notes taken during interviews to allow for understanding how the words were said or narrated during the interview [38]. Each transcript for each respondent was typed as a Microsoft Word document and analyzed thematically as shown in the results chapter.

\subsubsection{Analysis}

The results of the study were subjected to thematic analysis. Thematic analysis was chosen because of its flexibility and its ability to focus on identifying and 
describing the implicit and explicit ideas within the data [39] [40]. Themes which capture something important about the data in relation to the research question were identified and similarly, patterned responses or meaning within the data set were themed in accordance with [41]. Care was taken to ensure that themes do not overlap and writing the interview questions as a theme was also avoided. Proper matching was done to avoid mismatching of theme and content of the data.

Based on the research question of exploring the health workers perspective on the effectiveness of DOTS in Kaduna state, Six Themes were identified and drawn out for analysis of the interview result. The themes were based on the basic cardinal of DOTS which includes detection of new case, effective laboratory services, uninterrupted supply of free drugs and Political and Government commitment. Themes and codes identified for the research were

Theme

1) Laboratory services

2) Drug supply

3) Suggestions on how to improve
Code

LS

DS

GI

\section{Results}

Themes and codes identified for the research were

1) Laboratory services

2) Drug supply

3) Suggestions on General improvement

GI

Key findings on each theme are presented in this result chapter, to ensure anonymity; the codes used during the interviews were maintained in situations where the relevant statement of respondents will be quoted. The letter $\mathrm{Z}$ denotes the zone where the respondent was interviewed while the letter $\mathrm{R}$ stands for respondent number in that particular zone, as such Z1R1 denotes respondent number 1 from Zone 1 and so on.

\subsection{Laboratory Services (LS)}

Effective and functional Laboratory is another cardinal principle of DOTS without which it will be practically impossible to run the centre. According to the results of the present study, all 18 respondent agree to the having a Laboratory in their respective centres and that it is an important component of DOTS,

Z3R2: ...the Lab is the eye of the hospital and each time we have any miss diagnosis in the Lab, usually everywhere is affected.

Z1R5: Laboratory services are very important because you can have TB suspect, if the laboratory is not good enough, you may not even have positive result and you are likely to miss the cases.

Z2R3: ...the laboratory is the backbone of $\mathrm{TB}$, without the Laboratory, there will be no detection of new cases, and the Laboratory detects new cases, the DOTS is effective, without that no DOTS. 
According to one of the respondent (Z1R5), their Laboratory has attained International Status and recognition and is one of the best in the country

The centre has achieved international recognition in this respect. Recently we had a team that come for international accreditation; so far in the country, we are about the best.

Z3R3: Actually the Lab they are performing well, they are doing well actually they are very successfully we appreciate the work that they are doing there, Yes.

The generalized problems associated with the Laboratories of the DOTS centre covered by the study ranges from shortage of reagents/materials epileptic or erratic supply of electricity. Despite having stand by generators in most of the centres, fuelling the generators is a major bottle neck.

Z2R3: Well, there was a time we had a little problem, I will say in 2011, early 2011 we had problem but the problem is not from the centre but it is from the control, there are some material we lack, like the sputum reagents, for almost three months and the hospital has to provide the reagents and we collected some token money from the patients which is not suppose to be, but now we have settled and everything is okay now.

Z3R4: Laboratory is functioning and everything is good, the only problem is lack of reagents sometimes.

Z3R3: so when they go to the lab and there is no light, we have to buy fuel for the test, and we ask them to pay 50-50 Naira to contribute for the lab people to buy fuel, they will say we are poor and cannot give the money.

Despite the Labs been doing well, some respondents believe there is still room for improvement. Z1R2 is of the opinion that if improvement will be made to the Lab or diagnostic techniques, it will be better for the centre.

Therefore, to some certain extent it is effective but if we can have a more improved method of diagnosis, it would be better method of diagnosis, it would be better.

\subsection{Drugs Supply}

Uninterrupted adequate drug supply is a most for any DOTS program. Been a directly observed therapy, patients are expected to visit the DOTS centre daily for their drugs, in cases where they live within a considerable distance, drugs are giving to religious or community leaders to ensure patients compliance.

During the course of the study, all respondents agreed unanimously that the sources of drugs supply to their respective centres was the Kaduna State Tuberculosis and leprosy control Program office from the state capital and that drugs are been supplied quarterly. Z1R3, who is a pharmacist further clarified the issue and traced the sources to the Abuja office of the National Program on Tuberculosis and Leprosy control where he stated 
The sources of drugs supply to the Centre is through the World Health Organisation (WHO) and through the Federal Ministry of Health in Nigeria.

Although most respondent were of the opinion that the quantity of the drugs is enough, almost all admitted having a shortage of drugs in early 2011. When asked what was the reason for the shortage, almost all respondents opined that it was a logistic problem of some kind, however, Z1R4 further clarifies the issue:

In the past, sometimes we would not receive supply of drugs in a quarter due to logistics problem. It was either we were told that the drugs were in the port, due to lack of transportation, or one thing or the other. Then, we used to have inadequate supply.

According to Z1R5 the shortages experienced in the centres was because they don't place orders before they exhaust their supply.

There were instance that we had shortages, like sometimes last year there was shortage, especially in some other centers, that some of the staff, as you know you learn from experience, they do not request before they drug they have in stock is exhausted. They only request when they have, may be one or two kits; just few kits. So when they are requisition, they run short of drugs. Meanwhile the drugs were in the store so it's here we request when we have an average of Six Month supply, we start requesting.

Contrary to all the other respondents who were of the opinion that the drug supply is adequate, was the assertion of Z1R2 who stated that the quality of the drugs is okay but the quantity is not enough

In terms of quality it is okay, but the quantity is the problem because most of the times we run out of the drugs, and that affects the dose of those that are already on the drugs. Once you start the drugs and you stop, it gives room for resistant strength of the tuberculosis these days, and if there is a way to overcome help to eliminate its resistant strain.

Z1R6 also corroborate this where he stated that he is not satisfied with the drug supply and that it is not adequate, according to him

Honestly the sources is mainly from the federal Government, to be candid I am not satisfied because in instances whereby we are always having OS; OS.

The Tuberculosis drugs been of two categories, according to a respondent, they do not have problem with the category 1 drugs, however, cat 2 is in short supply and they find it difficult to get supply of cat 2 .

Yes is enough, category 1 but category 2 there is problem, most of the patients have to be coming up and down to get the drug.

\subsection{Improvement}

Generally this is the area where respondents differed in their respective Submis- 
sions, due largely to individual differences of their respective centres. While some were of the opinion that improving manpower is the best way to improve the services of DOTS, others opined that giving the health workers some incentives as motivation is the best way to achieve effectiveness.

Z3R2: I would have wish there will be special incentives for those that are working in the DOTS clinic in form of morale booster like gift or anything cash to a kind of improve their services to make them committed, you know when you give somebody something he can do extra so I wish something like that should have come up.

Z2R6: Like may be those that are taking care of HIV and TB will have extra allowances besides their salaries... yes, it will improve.

According to one respondent, community awareness which can be achieved through community sensitisation is the best way to improve the DOTS services in the state.

Z2R1: Health education to the people, I have to educate the people about tuberculosis and how dangerous it is and they have to know that it is curable so that they don't hide their status.

Z2R3: Community awareness comes first then drugs come second. Why I say community awareness is because most people they don't know that if they have cough they don't have to come here, we have the problem of prescribing drugs ourselves in Nigeria, people will be coughing and they will just go to chemist and buy drugs.

Other issues raised by the respondents as ways through which the services of DOTS can be improved in the state includes effective Laboratory, Conducive atmosphere for working and improvement in man power in the centres:

Z3R2: I will give priority to the Lab because the Lab is the eye of the hospital and each time we have any miss diagnosis in the Lab, usually everywhere is affected.

Z1R6: The CHEW (Health worker) needs a good environment to sit down to be able to attend to his patient... a conducive working environment for the parties involved in the work.

Z3R5: As I mentioned if we have more hands in the centre, the work will be better and patients will be taking their drugs anytime they come, they don't have to wait, so increase in manpower will definitely improve the system.

\section{Discussion}

\subsection{Drug Supply}

Regular and uninterrupted drugs supply is a most for any DOTS system to succeed [41]. During the course of the research, it was discovered that the sources of 
drug supply to all the centres was from National TB and Leprosy control program office of Kaduna state. Also the drugs are supplied regularly except for an occasional shortage witnessed in early 2001 . The drugs are of required quality and sufficient. The shortage of the drugs was attributed to logistic problems mostly of transportation in nature. There is therefore the need for operational framework to strengthened TB drug supply and management to reduce the incidence of shortages [42] such as development of dedicated transport system for TB drugs should be done. In terms of quantity and quality of drugs used for TB control in the state, it is satisfactorily according to the perspective of the health workers explored. This contravenes the findings of [43] [44] where irregular supply of drugs is associated with DOTS services and was found to be hindering the performance of the program in Nigeria.

\subsection{Laboratory Services}

A functional laboratory is a basic requirement for tuberculosis control through the use of DOTS, during the course of the study, it was discovered that all centres had a functional Laboratory as advocated by the world Health Organisation [45]. Despite having Laboratories in all the centres, each centre has its own Problems hindering the performance of the Laboratory. In some DOTS centres the laboratory is understaffed with only one qualified hand manning the Tuberculosis bench while in some others, periodic shortage of reagents and slides were the problems. These findings are corroborated by the findings of [43] [46] [47] [48]. One identified general problem affecting almost all the centres as perceived by the respondents is unsteady supply of electricity, to this end, some centres have stand by generators however, and maintenance and fuelling the generators is proving difficult in most cases. By implication, all DOTS centres in Kaduna State have a functional laboratory, however, the aspect of sputum microscopy requiring electricity is suffering from non-steady supply of electricity.

\subsection{Suggestions on General Improvement}

During the course of the study, all respondents came up with suggestions on how to improve the DOTS centres. A unanimous suggestion is equipping the laboratories with state of the art equipment as suggested by [45] as well as supplying drugs before the exhaustion of existing stock. More Government commitment has been suggested by the respondents during the study as possible ways of improving the DOTS services in Kaduna state, this has been suggested by earlier workers [41] [47]. Similarly, improved drugs supply has been suggested as a means of improving the DOTS services in Kaduna State. This has also been reported by other workers across the globe as Effective drugs supply is known to be an integral components of DOTS [43].

\subsection{Public Health Relevance, Conclusions and Recommendations}

The research generated data on drugs supply and effective laboratory services as 
they affect the performance of the DOTS centres or rather reducing their efficiencies towards tuberculosis control, which hitherto was not available, was generated through exploring the perspective of health care staff of the centres. when effectively utilized, the data will help in putting in place strategic measures that will bring about an improvement in the DOTS services across the state thereby reducing the incidence of Tuberculosis in Kaduna state and Nigeria at large.

Some of the recommendations based on the research findings on best ways to improve the DOTS services in Kaduna state include:

1) Heath Education of the Populace

The results of this study found that a lot of patients don't know about the existence or services rendered by DOTS, this is corroborated by the findings of [43] who studied the challenges hindering the performance of DOTS in Ilorin Nigeria and [49] in Russia. Educating the populace on the causes, symptoms and health effects of tuberculosis as well as its treatment will help in increasing new case detection [48]. Although in most of the centres posters on Tuberculosis and its control measures were pasted, the electronic media such as the radio is more popular with the local population and should be used to reach the communities. Good delivery methods of messages should be developed in accordance with the different socio cultural groups in the state based on geographical locations to be aired regularly.

2) Development of Baseline Data

Currently data on Tuberculosis epidemiology in Kaduna state is scanty, for effective control program, there is the need for a baseline studies to be conducted to come up with compressive baseline data that will allow for comparison in the feature to see how good or otherwise the DOTS control program is fairing. This has been recommended by the National Centre for Tuberculosis and Leprosy control centre, Zaria in its 2008 annual report [48].

3) Integrating the Control with HIV/AIDS

The emergence of HIV/AIDS was complemented by a surge in cases of Tuberculosis, from the present study, some TB patients were found to be co infected with HIV/AIDS and in such cases, it is better to merge the treatment together [47] [50], it has been reported by health workers in south Africa that HIV/TB co infected patients tends to take their HIV status more important than their TB status [51], this therefore makes the merger of the two treatment necessary.

4) More encouragement for private clinics to get involved in the DOTS and TB control in the state. The existing private clinic partnership in the state should be further strengthened as recommended by earlier workers [11].

5) Further research should be conducted to determine the perspective of the patients attending the DOTS centres on the possible reasons of defaulting and how best to improve the services of the DOTS centres in the State, as the patients' perspective is equally very important [52] as they are major stake holders in the control program.

Although Tuberculosis is a worldwide problem, Nigeria is the fifth most affected nation [21] therefore there is the need to strengthened the DOTS system 
and ensure effective control of the disease, similarly the Government should make the entire health system affordable, equitable, accessible, sustainable and of good quality [52].

\section{References}

[1] WHO (2010) World Health Organisation 2010 Tuberculosis Factsheet. http://www.who.int/mediacentre/factsheets/fs104/en/

[2] Martins, N. (2008) Qualitative Study of Barriers to and Enabling Factors for Tuberculosis Treatment Adherence in Timor Leste. Public Lecture University of Sydney. http://sydney.edu.au/global-health/images/content/news/TB_Presentantion.pdf

[3] Obermeyer, Z., Abbott-Klafter, J. Christopher, J. and Murray, L. (2008) Has the DOTS Strategy Improved Case Finding or Treatment Success? An Empirical Assessment. PLoS ONE, 3, e1721. https://doi.org/10.1371/journal.pone.0001721

[4] World Health Organisation Report (2004) Global Report on Tuberculosis. http://www.who.int/tb/publications/global_report/archive/en/

[5] Itah, A.Y. and Udofia, S.M. (2005) Epidemiology and Endemicity of Pulmonary Tuberculosis (PTB) in Southeastern Nigeria. Southeast Asian Journal of Tropical Med Public Health, 36, 317-23. http://www.ncbi.nlm.nih.gov/pubmed/15916036

[6] Cosivi, O., Grange, J.M., Daborn, C.J., Raviglione, M.C., Fujikura, T., Cousins, D., Robinson, R.A., Huchzermeyer, H.F.A.K.., de Kantor, I. and Meslin, F.X. (1998) Zoonotic Tuberculosis due to Mycobacterium bovis in Developing Countries. Emerging Infectious Diseases, 4, 59-70. http://www.ncbi.nlm.nih.gov/pmc/articles/PMC2627667/pdf/9452399.pdf

[7] Murray, C.J.L., Styblo, K. and Rovillan, A. (1990) Tuberculosis in Developing Countries: Burdens, Interventions and Costs. Bulletin of the International Union against Tuberculosis and Lung Disease, 65, 6-24.

[8] Beith, A., Eichler, R and Weil, D. (2009) Worldwide: Incentives for Tuberculosis Diagnosis and Treatment. In: Eichler, R., Ed., Performance Incentives for Global Health: Potential and Pitfalls, Levine and the Working Group on Performance-Based Incentives, Center for Global Development, Washington DC.

[9] Ananthakrishan, R., Jeyaraj, A., Plani, G. and Sathiyasekaran, B.W.C. (2012) Socioeconomic Impact of TB on Patients Registered within RNTCP and Their Families in the Year 2007 in Chennai, India. Lung India, 29, 221-226.

http://www.lungindia.com/article.asp?issn=0970-2113;year $=2012$; volume $=29$;issue $=$ 3; spage $=221$; epage $=226$; aulast $=$ Ananthakrishnan

[10] Anonymous (2011) University of Liverpool Lecture Notes 2011.

[11] Gidado, M. and Ejembi, C.L. (2009) Tuberculosis Case Management and Treatment Outcome: Assessment of the Effectiveness of Public-Private Mix of Tuberculosis Programme in Kaduna State, Nigeria. Annals of African Medicine, 8, 25-31. http://www.annalsafrmed.org/text.asp?2009/8/1/25/55760 https://doi.org/10.4103/1596-3519.55760

[12] Awofeso, N. (1998) Implementing Tuberculosis Control Program in Kaduna State, Nigeria. International Journal of Tuberculosis and Lung Disease, 24, 336-337.

[13] USAID (2011) USAID/NIGERIA: HIV/AIDS and Tuberculosis. http://pdf.usaid.gov/pdf_docs/Pdacp977.pdf

[14] Atkinson, P., Coffey, A., Delamont, S., Lofland, J. and Lofland, L. (Eds.) (2001) Handbook of Ethnography. Sage, London. https://doi.org/10.4135/9781848608337 
[15] Griffiths, F. (1996) Qualitative Research: The Research Questions It Can Help Answer, the Methods It Uses, the Assumptions behind the Research Questions and What Influences the Direction of Research. A Summary of the Panel Discussion at the Conference "Exploring Qualitative Research in General Practice". Family Practice, 13, S27-S30.

[16] Samdahl, D.M. (1999) Epistemological and Methodological Issues in Leisure Studies. In: Jackson, E.J. and Burton, T.L., Eds., Leisure Studies: Prospects for the Twenty-First Century, Venture Publ., State College, 119-133.

[17] Marshall, N. (1996) Sampling Foe Qualitative Research. Family Practice, 13, 522-525. https://doi.org/10.1093/fampra/13.6.522

[18] Halcomb, E.J. and Andrew, S. (2005) Triangulation as a Method for Contemporary Nursing Research. Nurse Researcher, 13, 71-25.

[19] Anonymous (2006) National Population Census Publication. National Population Commission, Abuja.

[20] Nguyen, T.A., Oosterhoff, P., Pham, N.Y., Hardon, A. and Wright, P. (2009) Health Workers' View on Quality of Prevention of Mother-to-Child Transmission and Postnatal Care for HIV-Infected Women and Their Children. Human Resources for Health, 7, 39. http://www.human-resources-health.com/content/7/1/39 https://doi.org/10.1186/1478-4491-7-39

[21] WHO (2008) Global Tuberculosis Control-Surveillance, Planning and Financing. World Health Organisation, Geneva. http://www.who.int/tb/publications/global_report/en/

[22] Woolf, R., Salanipoli, F.M.L. and Kemp, J.R. (2006) Can District-Level Tuberculosis Case-Finding in Malawi Improved? A Qualitative Study of Health Worker Perspective in Dedza District. Malawi Medical Journal, 18, 66-71. http://www.ajol.info/index.php/mmj/article/view/10912/37706

[23] Suri, A., Gan, K. and Carpenter, S. (2007) Voices from the Field: Perspective from Community Health Workers on Health Care Delivery in Rural Kwazulu-Natal, South Africa. Journal of Infectious Diseases, 196, S505-S511.

http://jid.oxfordjournals.org/content/196/Supplement_3/S505.full https://doi.org/10.1086/521122

[24] Kvale, S. (1996) InterViews: An Introduction to Qualitative Research Interviewing. Sage, Thousand Oaks.

[25] Litva, A. and Jacoby, A. (2002) Qualitative Methods and Evidence-Based Practice. In: Craig, J. and Smyth, R., Eds., The Evidence Based Practice Manual for Nurses, Elsevier Science, Edinburgh, 136-163.

[26] Hill, C.E., Knox, S., Thompson, B.J., Williams, E.N., Hess, S.A. and Ladany, N. (2005) Consensual Qualitative Research: An Update. Journal of Counseling Psychology, 52, 196-205. http://tcp.sagepub.com/content/35/2/209.abstract https://doi.org/10.1037/0022-0167.52.2.196

[27] Turner, D.W. (2010) Qualitative Interview Design: A Practical Guide for Novice Investigators. The Qualitative Report, 15, 754-760. http://www.nova.edu/ssss/QR/QR15-3/qid.pdf

[28] Boyce, C. and Neale, P. (2006) Conducting In-Depth Interviews: A Guide for Designing and Conducting In-Depth Interviews for Evaluation. http://www2.pathfinder.org/site/DocServer/m_e_tool_series_indepth_interviews.pdf

[29] McNamara, C. (2009) General Guidelines for Conducting Interviews. http://managementhelp.org/evaluatn/intrview.htm 
[30] Pope, C. and Mays, N. (1995) Qualitative Research: Reaching the Parts Other Methods Cannot Reach: An Introduction to Qualitative Methods in Health and Health Services Research. British Medical Journal, 311, 42-45.

[31] Anonymous (2009) What Is Ethics? Markkula Centre for Applied Ethics, Santa Clara University, Santa Clara. http://www.scu.edu/ethics/practicing/decision/whatisethics.html

[32] Catherine Pope and Nick Mays (1995) Qualitative Research: Reaching the Parts Other Methods Cannot Reach: An Introduction to Qualitative Methods in Health and Health Services Research. BMJ, 311, 42-45.

[33] Richards, H.M. and Schwarz, L.J. (2002) Ethics of Qualitative Research: Are There Special Issues for Health Service Research? Family Practice, 19, 135-139.

[34] Green, J. and Thorogood, N. (2009) Qualitative Methods for Health Research. 2nd Edition, Sage, London.

[35] Anonymous ( 2011) University of Liverpool Lecture Notes 2011.

[36] Halcomb, E.J. and Davidson, P.M. (2006) Is Verbatim Transcription of Interview Data Always Necessary? Applied Nursing Research, 19, 38-42. https://doi.org/10.1016/j.apnr.2005.06.001

[37] MacLean, L.M., Meyer, M. and Estable, A. (2004) Improving Accuracy of Transcripts in Qualitative Research. Qualitative Health Research, 14, 113-123. https://doi.org/10.1177/1049732303259804

[38] Wengraf, T. (2001) Qualitative Research Interviewing: Biographic Narrative and Semi-Structured Methods. Sage Publications, London, 7. https://doi.org/10.4135/9781849209717

[39] Bailey, J. (2008) First Steps in Qualitative Data Analysis: Transcribing. http://fampra.oxfordjournals.org/

[40] Braun, V. and Clarke, V. (2006) Using Thematic Analysis in Psychology. Qualitative Research in Psychology, 3, 77-101. https://doi.org/10.1191/1478088706qp063oa

[41] Ilodigwe, E.E. (2002) Optimising Directly Observed Treatment, Short-Course Strategy in Nigeria. Nigerian Quarterly Journal of Hospital Medicine, 13, 36-39.

[42] WHO (2004) Global Tuberculosis Control, Surveillence, Planning, Financing. World Health Organisation Report.

http://www.who.int/tb/publications/global_report/archive/en/

[43] Bello, S.I. (2010) Challenges of DOTS Implementation Strategy in the Treatment of Tuberculosis in a Tertiary Health Institution, Ilorin, Nigeria. African Journal of Pharmacy and Pharmacology, 4, 158-164. http://www.academicjournals.org/ajpp

[44] Akanbi, M.O. and Ukoli, C.O. (2009) Are Weak Health Systems a Brewing Ground for Multi-Drug-Resistant Tuberculosis? African Journal of Respiratory Medicine, 5, $21-22$.

http://www.africanjournalofrespiratorymedicine.com/articles/september_2009/Are \%20weak\%20health\%20systems\%20a\%20brewing\%20ground $\% 20$ for $\% 20$ multi-drug -resistant\%20tuberculosis.pdf

[45] WHO (2009) World Health Organization, Stop TB Partnership Retooling Task Force, Stop TB Partnership New Diagnostics Working Group. New Laboratory Diagnostic Tools for Tuberculosis Control. http://www.stoptb.org/wg/new_diagnostics/

[46] Erah, P.O. and Ojieabu, W.A. (2009) Success of the Control of Tuberculosis in Nigeria: A Review. International Journal of Health Research, 2, 3-14.

https://www.ajol.info/index.php/ijhr/issue/view/7340 
[47] NTBL (2008) Annual Reports of NTBL Centre Zaria.

http://www.ntbltc.org/reports/annual\%20report\%20NTBLTC\%20Zaria\%202008.pdf

[48] Dimitrova, B., Balabanova, D., Atun, R., Drobieweski, F., Levichiva, V. and Cooker, R. (2006) Health Service Providers' Perceptions of Barriers to Tuberculosis Care in Russia. Health Policy Plan, 21, 265-274.

http://www.ncbi.nlm.nih.gov/pubmed/16728512

https://doi.org/10.1093/heapol/czl014

[49] Ige, O.M. and Oladukun, R.E. (2011) Treatment Outcome of Newly Diagnosed Sputum Positive Adult Tuberculosis Cases in the Context of HIV Infection. Journal of Infectious Disease and Immunity, 3, 210-217.

http://www.academicjournals.org/JIDI

[50] Naidoo, P. (2009) Public Health Care Practitioner's Reflection on Tuberculosis Patients Perspectives on Factors Influencing Their Adherence to the Directly Observed Treatment Shortcourse. The Open Public Health Journal, 2, 33-38.

[51] Khan, M.A., Walley, J.D., Witter, S.N., Imran, A. and Safdar, N. (2002) Cost and Cost-Effectiveness for the Different DOTS Strategy for the Treatment of Tuberculosis in Pakistan. Health Policy Plan, 17, 178-186.

http://heapol.oxfordjournals.org/content/17/2/178.short

https://doi.org/10.1093/heapol/17.2.178

[52] Ajay, T., Christopher, J., Murray, L., Jeremy, A.L. and David, B.E. (2000) Measuring Overall Health System Performance for 191 Countries. GPE Discussion Paper Series No. 30, EIP/GPE/EQC World Health Organization.

http://www.who.int/healthinfo/paper30.pdf 